\title{
Mutational Analysis of DNASE I Gene in Diabetic Patients
}

Hateem Zafar Kayani, Nageen Hussain

Department of Microbiology and Molecular Genetics, Quaid-e-Azam Campus, University of the Punjab,

Number of figures: 04

Lahore, Pakistan.

Number of tables: 03

Corresponding author:

\section{Dr. Nageen Hussain}

Assistant Professor,Department of Microbiology and Molecular Genetics, Quaid-e-Azam Campus, University of the Punjab, Lahore, Pakistan.

Email: nageen1704@hotmail.com, phone number: 0321-4736630.

\begin{abstract}
The main aim is to analyze the mutations of DNASE I gene in diabetic patients. A total of 120 diabetes patients and 120 controls were sampled. The total number of male diabetic patients included in the study was 79 (66\%) while female patients were 41 (34\%) in number. Exon 8 of the DNASE I gene was amplified by using thermocycler. The possible band of interest was located at 165 base pairs. Two samples showed similar missense mutations at $127^{\text {th }}$ position of exon 8 which replaced amino acid Arginine (Arg) to Glutamine (Gln). All controls showed no mutations. The association of diabetes with different levels of blood pressure and body mass index (BMI) were found to be significant.
\end{abstract}

Keywords- Deoxyribonuclease I; Polymerase Chain Reaction; Insulin-Dependent Diabetes Mellitus; Non-Insulin Dependent Diabetes Mellitus.

\section{Council for Innovative Research}

Peer Review Research Publishing System

\section{Journal: JOURNAL OF ADVANCES IN BIOTECHNOLOGY}

Vol. 3, No 3.

www.cirjbt.org , jbteditor@gmail.com 


\title{
Introduction
}

Diabetes mellitus (DM) belongs to a group of metabolic diseases in which blood sugar levels rise in a patient. The symptoms of disease include frequent urination, increased thirst, and increased hunger. Untreated diabetes can have severe complications, including heart disease, kidney failure, and damage to the eyes. Diabetes is caused due to two main reasons; either the pancreas lack insulin production or the response to the insulin production is deficient. There are two main types of diabetes mellitus. Type 1 Diabetes results from the body's malfunction to produce insulin. This type was earlier known as "insulin-dependent diabetes mellitus" (IDDM) or "juvenile diabetes". Type 2 Diabetes results from insulin resistance, a condition in which cells fail to use insulin properly, sometimes also with an absolute insulin deficiency. Type 2 diabetes was formerly recognized as "non insulin-dependent diabetes mellitus" (NIDDM) or "adult-onset diabetes" (Rosenbloom et al., 1999).

Deoxyribonuclease I (DNase I), is an endonuclease coded by the human gene DNASE I. DNase I is a nuclease that cleaves DNA preferentially at phosphodiester linkages adjacent to a pyrimidine nucleotide, yielding 5'-phosphateterminated polynucleotides with a free hydroxyl group at position 3', on average producing tetranucleotides. It acts on single-stranded DNA, double-stranded DNA, and chromatin. A single nucleotide mutation usually decreases the DNase I activity by $10-40 \%$. The enzyme is a simulated glycoprotein with a molecular mass of about 38,000 Daltons. DNASE I gene is approximately $3.2 \mathrm{~kb}$ long with 9 exons separated by 8 introns.. Polymerase chain reaction (PCR) was performed using DNA extracted from a panel of cloned human/rodent hybrid cell lines carrying different human chromosomes. They assigned the DNASE1 gene to human chromosome 16. Furthermore, regional localization to $16 \mathrm{p} 13.3$ was performed by PCR analysis of a high-resolution mouse/human somatic cell hybrid panel that contained defined portions of human chromosome 16 (Yashuda et al., 1995).

Recent studies in Pakistan reported that the prevalence of newly diagnosed cases of diabetes was $5.1 \%$ in males and $6.8 \%$ in females who are living in the urban areas and the prevalence of people living in rural areas was $5.0 \%$ in males and $4.8 \%$ in females (Aziz et al., 2009). The other important complication of diabetes is the renal failure, also known as diabetic nephropathy. There is an elevated risk of about four times of renal failure in diabetic patients as compared to normal healthy people. Some cases were also reported about the linkage of diabetes to blindness. The risk of developing ophthalmologic complications is 3 times as compared to normal individuals and complication like neuropathy occurs in $50 \%$ of diabetic patients which leads to muscle weakness, pain and other problems (Ringborg et al., 2008).

The main objective of the study was to identify the mutation site in the DNase I gene in diabetic patients. The main focus of the study was exon 8 which has some known mutations.

\section{MATERIALS AND METHODS:}

A total of 120 diabetic patients and 120 controls were enrolled in this study. Completely normal, healthy individuals were considered as controls. Blood samples from 120 diabetic subjects were collected in EDTA coated vacutainers and written informed consent form was received from each subject. Genomic DNA was extracted from all blood samples by using proteinase $\mathrm{K}$ method. The DNASE I gene mutations were assayed using thermocycler. The reaction mixture (50 $\mu \mathrm{l} /$ reaction) contained $25 \mu \mathrm{l}$ of the $2 \mathrm{X}$ PCR mix, template DNA $2.0 \mu \mathrm{l}$, primer mixture $1.0 \mu \mathrm{l}$ and nuclease free water in quantity of $22 \mu \mathrm{l}$. To amplify the exonic region, simple PCR program was used for 35 cycles using 96 wells thermal cycler. The annealing temperature was optimized by varying the temperature from $55^{\circ} \mathrm{C}$ to $58^{\circ} \mathrm{C}$. PCR primers were optimized at temperature of $56^{\circ} \mathrm{C}$. Later on, the PCR product was detected by using $1 \%$ agarose gel and DNA ladder (Fermentas, USA) was used to detect the banding pattern. Univariate analysis and chi square test were also applied.

The PCR products were sent to Macrogen Company in Korea for sequencing. Sanger sequencing is a method of DNA sequencing based on the selective incorporation of chain terminating dideoxynucleotides by DNA polymerase during in vitro DNA replication. The sequenced samples were initially checked by CAP 3 software and then the NCBI software confirmed the similarity between sequenced samples. DNA star was used to translate these sequenced DNA and mutations were analyzed in EditSeq. The codon changes were noted and amino acid changes were studied.

The region of "DNASE gene exon 8" that was amplified is as follows: (NCBI database

\begin{abstract}
"TGCACTGGCAGGTCCCAGGGCTCTTAGTTTAGTTCCTGCGGGTGCTGAGCCAGGCCCATGTGTGAAAGGGGAAC CTACTTTCTCTTCCCAACACCCATCAGGATCGTGGTTGCAGGGATGCTGCTCCGAGGCGCCGTTGTTCCCGACTCG GCTCTTCCCTTTAACTTCCAGGCTGCCTATGGCCTGAGTGACCAACTGGTATGTGTCCTCCCTTGCACAGCCACATG AGGATGGGCACAGGAGCTCAGGTAGGCTCAGCCCAGACCCTGTGCCCACTTGCCTGCAGGCCCAAGCCAT"
\end{abstract}

The set of primers amplified the 165 base pair sequence which was the band of interest identified through the DNA ladder on the agarose gel. The full sequence is shown below.

\begin{abstract}
"TTCTCTTCCCAACACCCATCAGGATCGTGGTTGCAGGGATGCTGCTCCGAGGCGCCGTTGTTCCCGACTCGGCTC TTCCCTTTAACTTCCAGGCTGCCTATGGCCTGAGTGACCAACTGGTATGTGTCCTCCCTTGCACAGCCACATGAGG ATGGGACACAGGAG"
\end{abstract}




\section{RESULTS:}

A total of 120 diabetic patients and 120 controls were enrolled in this study. Completely normal, healthy individuals were considered as controls. The total number of male diabetic patients included in the study was $79(66 \%)$ while female patients were $41(34 \%)$ in number. Of the 79 male diabetic patients, 74 (94\%) patients were diagnosed with more common diabetes type 2 while $5(6 \%)$ patients were diagnosed with the rare diabetes type 1 . The total number of female diabetic patients was 41 . Out of these 41 diabetic females, 36 (88\%) females were patients of type 2 diabetes and 5 (12\%) females were diagnosed cases of type 1 diabetes. The association of diabetes type 1 and type 2 was also checked for other disorders like hypertension (HT), cardiovascular diseases (CVD), chronic kidney disorder (CKD) and respiratory distress (RD) through different international standards and guidelines. Figure 4 and 5 shows the association of diabetes type 1 and type 2 with other disorders.

The ten samples which were sequenced and analyzed belonged to 10 different categories based on the associated diseases with diabetes. Sample one was standard control. Samples 2, 3 and 4 belonged to diabetes type 1 and its associated diseases. Samples 5 to 10 represented diabetes type 2 with its associated disorders. Figure 1 shows a missense mutation of $G>A$ at $127^{\text {th }}$ position of the exon 8 in DNASE gene. In genetics, a missense mutation is a point mutation in which a single nucleotide change results in a codon that code for a different amino acid. There was a missense mutation found in sample 2 and sample 6 with both mutations coding for Glutamine (GIn) instead of Arginine (Arg). There was no relevant study which could indicate any relationship of DNase I with diabetes type 1 or type 2 although this mutation is discussed with reference to structural changes in DNase I. The amino acid sequence of the exon 8 in the DNASE I gene is explained in figure 2.

250

* 260

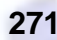

Phenotype (sample 2): ATG CTG CTC CAA GGC GCC GTT Met Leu Leu Gln Gly Ala Val

117 * 127 138

Phenotype (exon 8): ATG CTG CTC CGA GGC GCC GTT Met Leu Leu Arg Gly Ala Val

Figure 1: Substitution of $\mathrm{G}>\mathrm{A}$ shown at the 127th position of the exon 8 in DNASE / gene

"agG ATC GTG GTT GCA GGG ATG CTG CTC CGA GGC GCC GTT GTT CCC g Ile Val Val Ala Gly Met Leu Leu Arg Gly Ala Val Val Pro GAC TCG GCT CTT CCC TTT AAC TTC CAG GCT GCC TAT GGC CTG AGT Asp Ser Ala Leu Pro Phe Asn Phe Gln Ala Ala Tyr Gly Leu Ser

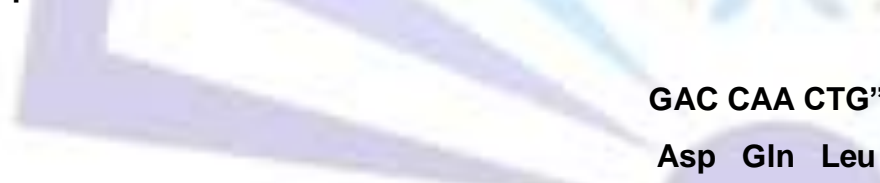

Figure 2: The amino acid sequence of the exon 8 in the DNASE I gene 


\section{ISSN 2348-6201}

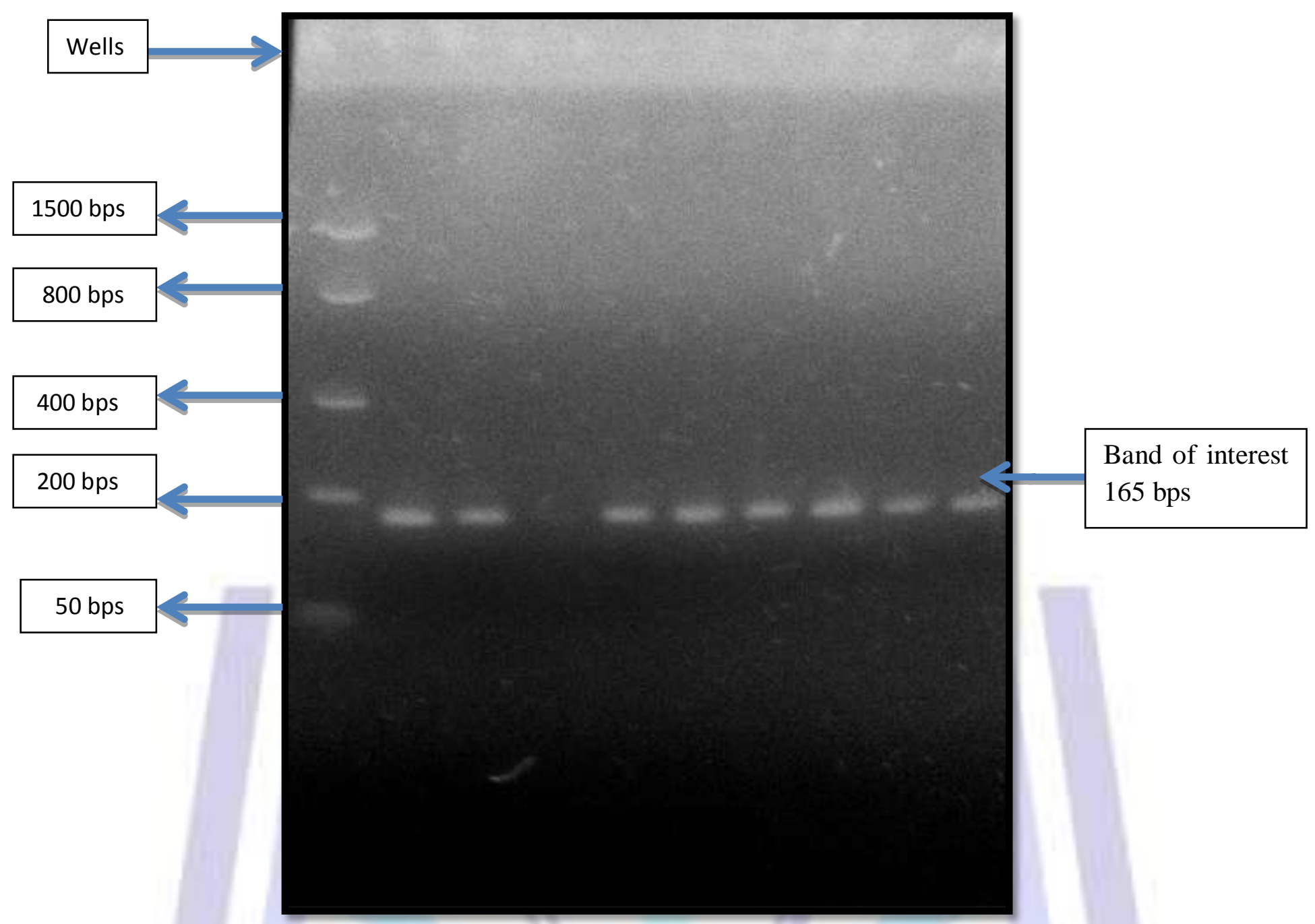

Figure 3: Labeled gel picture having desired PCR products bands at 165 base pairs along with low DNA ladder

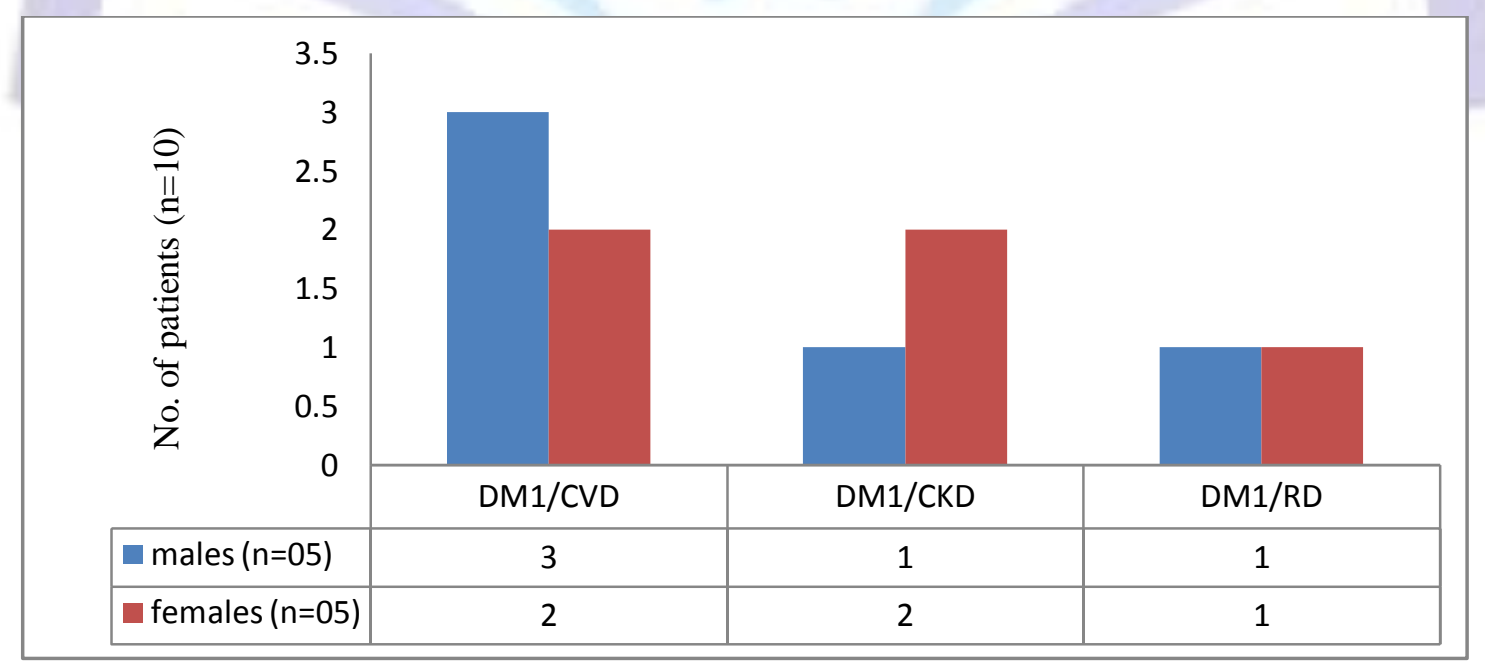

Figure 4: Diabetes type 1 with other diseases (KEY: DM1/CVD = Diabetes Melitus type 1/ Cardiovascular Diseases; DM1/CKD = Diabetes Melitus type 1/Chronic Kidney Diseases; DM1/RD = Diabetes Melitus type 1/Respiratory Distress) 


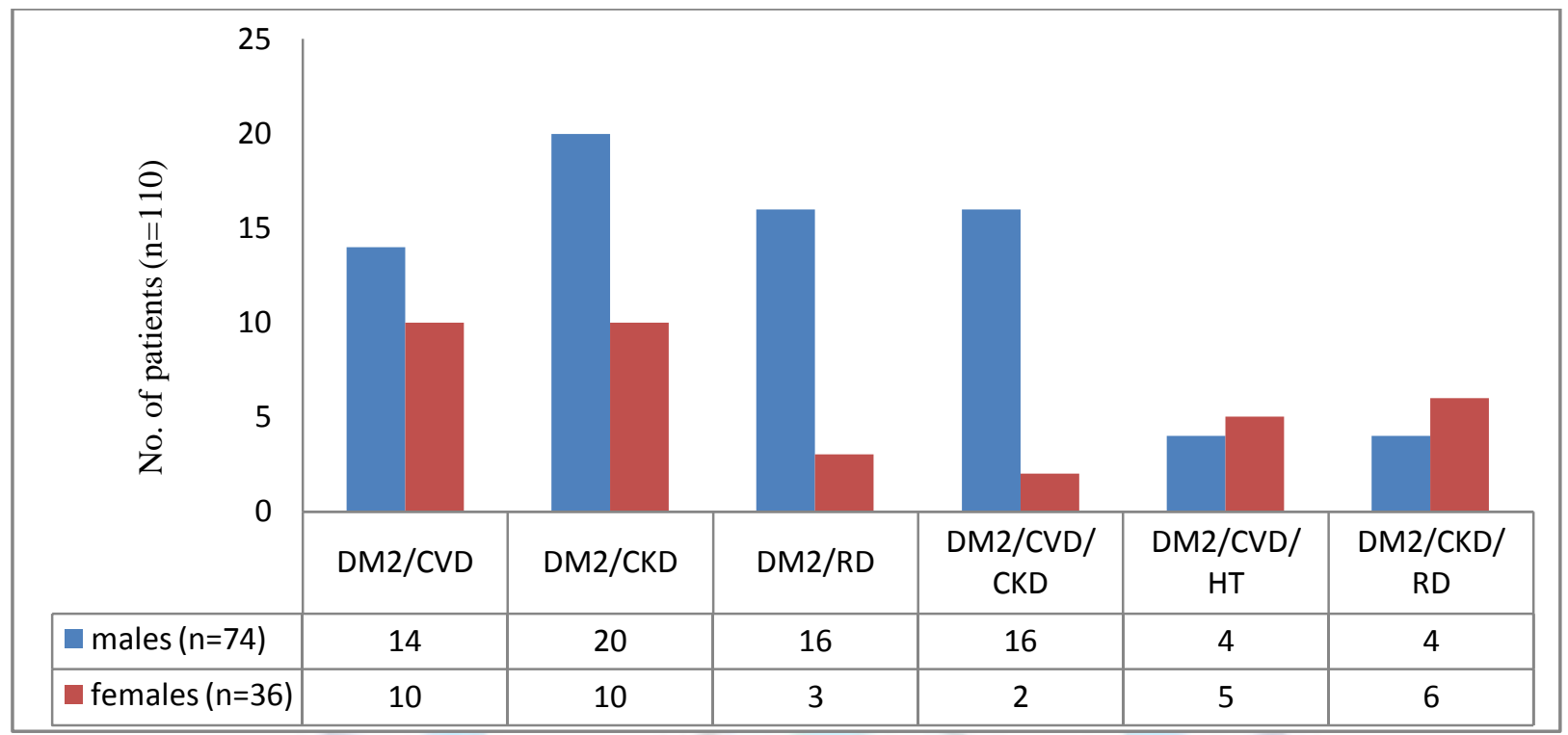

Figure 5: Diabetes, type 2 with other diseases (KEY: DM2/CVD = Diabetes Melitus type 2/ Cardiovascular Diseases; DM2/CKD = Diabetes Melitus type 2/Chronic Kidney Diseases; DM2/RD = Diabetes Melitus type 2/Respiratory Distress; DM2/CVD/CKD = Diabetes Melitus type 2/ Cardiovascular Diseases/ Chronic Kidney Diseases; DM2/CVD/HT = Diabetes Melitus type 2/ Cardiovascular Diseases/Hypertension; DM2/CKD/RD = Diabetes Melitus type 2/Chronic Kidney Diseases/ Respiratory Distress)

\section{DISCUSSION:}

Aging, obesity and insufficient energy consumption are independent risk factors of pathogenesis of type 2 diabetes. Obesity is directly linked with a decrease in muscle mass, induces insulin resistance and is strongly linked with the rapid increase in the number of middle and high aged patients. Even mild obesity (Body mass index (BMI) <25) causes a 4 to 5 fold increase in risk of developing diabetes (Barr et al., 2007). Chi square test was applied to find any significant association between BMI and diabetes. There was a significant association in this relation as the $P$ value was less than 0.05 (table 1). It can be concluded that BMI has direct influence on diabetes. Table 1 shows the association between BMI and diabetes. The variations in the association of diabetes with BMI may be either due to varied ethnicity or the various other genetic and environmental factors implicated in the regulation of blood sugar levels (Michael et al., 2014). The etiology of primary hypertension is unknown; however, its diverse hemodynamic and pathophysiologic derangements are unlikely to result from a single cause. Heredity is one of the main predisposing factors, but the exact mechanism is unclear. It is observed that environmental factors only influence the instances of hypertension in diabetic patients who are genetically susceptible. Obesity is also a major risk factor for diabetes and is also linked to hypertension (Aziz et al., 2009). There was a significant association between diabetes and blood pressure as shown in table 2 .

The request for accession numbers was made in the NCBI databases using the software Bank it. Several vector similarity sequences were edited through Vector Screen software. Some internal stop codons were also removed. NCBI blast services were also used to find $100 \%$ similarity with DNASE I gene. The code for the samples was "Banklt 1728978". Accession numbers are shown in table 3 . This is the first study of its kind which explains mutational analysis of DNASE I gene in diabetic patients. However, association of cardiovascular disorders and chronic kidney disorders might be critical in explaining the missense mutation in diabetes.

Table 1: Association between diabetes and BMI

\begin{tabular}{|l|c|c|c|c|}
\hline \multirow{2}{*}{ Diabetes } & \multicolumn{3}{|c|}{ Body mass index (BMI) } & P value \\
\hline & Underweight & Normal weight & Overweight & \\
\hline Males $(n=79)$ & 10 & 24 & 45 & 0.0344 \\
Females $(n=41)$ & 08 & 20 & 13 & \\
\hline
\end{tabular}


Table 2: Association between diabetes and different stages of blood pressure

\begin{tabular}{|c|c|c|c|c|}
\hline \multirow{2}{*}{ Diabetes } & \multicolumn{3}{|c|}{ Affection status of Hypertension } & P value \\
\hline & Hypertensive & Normotensive & Pre-hypertensive & \\
\hline Males $(\mathrm{n}=79)$ & 20 & 50 & 09 & 0.045 \\
Females $(\mathrm{n}=41)$ & 11 & 20 & 10 & \\
\hline
\end{tabular}

Table 3: Accession numbers of samples submitted in NCBI databases

\begin{tabular}{|c|c|}
\hline Sample No. & Accession numbers \\
& KJ862263 \\
\hline SEQ1 & KJ862264 \\
\hline SEQ2 & KJ862265 \\
\hline SEQ4 & KJ862266 \\
\hline SEQ5 & KJ862267 \\
\hline SEQ6 & KJ862268 \\
\hline SEQ7 & KJ862269 \\
\hline SEQ8 & KJ862270 \\
\hline SEQ9 & KJ862271 \\
\hline SEQ10 & KJ862272 \\
\hline
\end{tabular}

\section{REFERENCES:}

[1] Aziz, S., Noorulain, W., Zaidi, U. R., Hossain, K., Siddiqui, I. A., Prevalence of overweight and obesity among children and adolescents of affluent schools in Karachi. J Pak Med Assoc. 2009, 59 (1), 35-38.

[2] Barr, E.L., Zimmet, P.Z., Welborn, T.A., Jolley, D., Magliano, D.J., Dunstan, D.W., Cameron, A.J., Dwyer, T., Taylor, H.R., Tonkin, A.M., Wong, T.Y., McNeil, J., Shaw, J.E., Risk of cardiovascular and all-cause mortality in individuals with diabetes mellitus, impaired fasting glucose, and impaired glucose tolerance: the Australian Diabetes, Obesity, and Lifestyle Study. Circulation. 2007,116 (2), 151-157.

[3] Ringborg, A., Lindgren, P., Martinell, M., Yin, D.D., Schon, S., Stalhammar, J., Prevalence and incidence of Type 2 diabetes and its complications 1996-2003-estimates from a Swedish population-based study. Diabetes Medicine. 2008, 25 (10), 1178-1186.

[4] Rosenbloom, A.L., Joe, J.R., Young, R.S., Winter, W.E., Emerging epidemic of type 2 diabetes in youth. Diabetes Care. 1999, 22 (2), 345-354.

[5] Yasuda, T., Kishi, K., Yanagawa, Y., Yoshida, A., Structure of the human deoxyribonuclease I (DNase I) gene: identification of the nucleotide substitution that generates its classicalgenetic polymorphism. Annual Human Genetics. 1995, 59, 1-15. 